\title{
Stand der Arbeiten in der tripartiten Kooperation FMH / H+ / MTK
}

\author{
Roger Scherrer a, \\ Irène Marty ${ }^{b}$ \\ a FMH, Leiter Ressort \\ Ambulante Tarife und \\ Verträge Schweiz \\ b FMH, Stv. Leiterin Ressort \\ Ambulante Tarife und \\ Verträge Schweiz
}

Korrespondenz:

FMH / Ressort Ambulante Tarife und Verträge Schweiz

Froburgstrasse 15

CH-4600 Olten

Tel. 0313591230

Fax 0313591238

tarife.ambulant[at]fmh.ch
Die vorgesehenen linearen Kürzungen bei verschiedenen TARMED-Kapiteln, die der Bundesrat mittels seiner subsidiären Kompetenz in diesem Jahr vornehmen will, dürften die bestehenden Verzerrungen bei der Bewertung der einzelnen medizinischen Leistungen noch verstärken. Gemäss Erläuterungen zum entsprechenden Verordnungsentwurf vom Dezember 2013 ist vorgesehen, dass diese Eingriffe befristet bis zur Gesamtrevision der Tarifstruktur TARMED durch die Tarifpartner gelten sollen.

Zentral für die FMH ist, dass die Arbeiten zur TARMED-Gesamtrevision zügig vorankommen und möglichst bald eine gesamtheitlich revidierte Version der Tarifstruktur zur Verfügung steht, welche die Bewertung medizinischer Einzelleistungen wieder durch klare und transparente Mechanismen sicherstellt. Deshalb laufen die gemeinsamen Revisionsarbeiten von FMH, H+ und MTK (Tripartite Kooperation) auf Hochtouren weiter. Nachfolgend wird der aktuelle Stand dieser Revisionsarbeiten näher aufgezeigt.

\section{Abbildung 1}

Die tripartite Kooperation ist auf drei Ebenen organisiert.

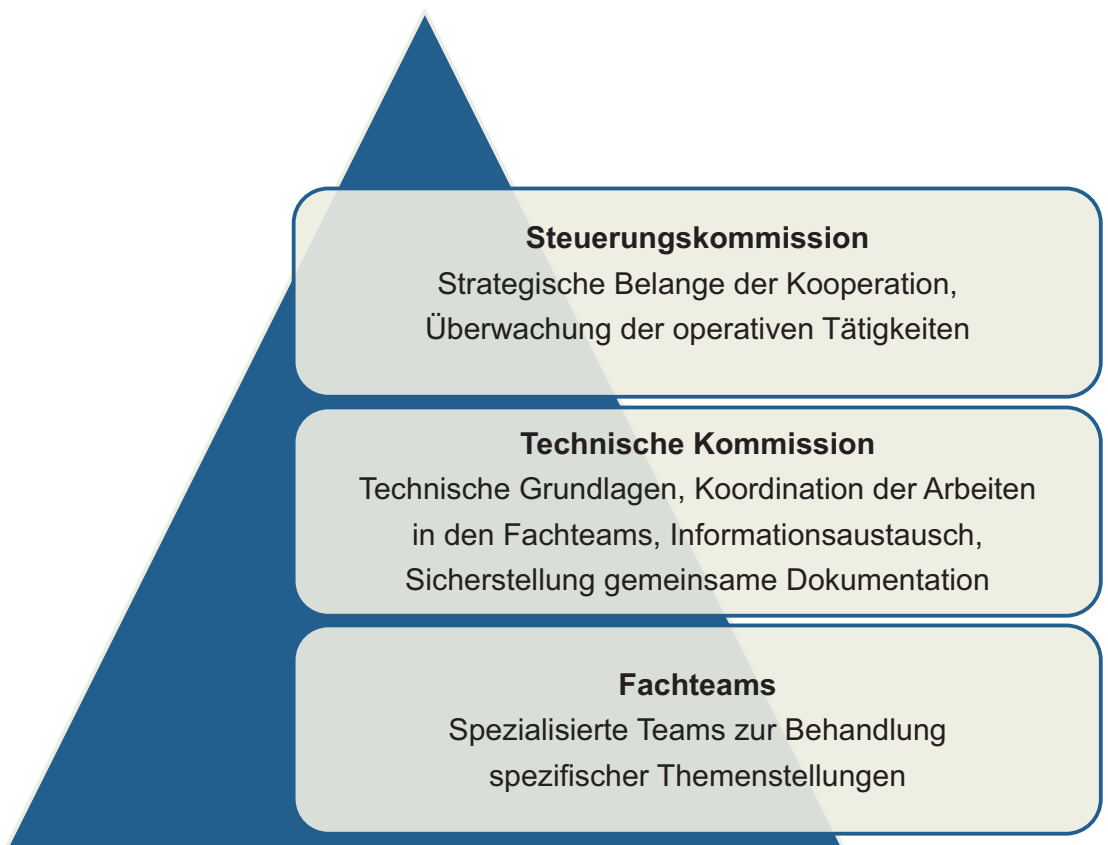

\section{Schwerpunkte der Tripartiten Kooperation FMH / H+ / MTK}

Die FMH begann mit der Revision der Tarifstruktur bereits Ende 2010. Seit Herbst 2012 arbeitet die Tripartite Kooperation, bestehend aus der FMH, $\mathrm{H}+$ und der MTK, mit vereinten Kräften an der Gesamtrevision des TARMED.

In der entsprechenden Absichtserklärung ist das gemeinsame Ziel klar festgehalten: «die gesetzeskonforme, betriebswirtschaftlich korrekte, aktualisierte und sachgerechte Bewertung der Leistungen in Arztpraxen, Spitälern und Kliniken, wenn immer möglich gestützt auf daten- und faktenbasierte Grundlagen.»

Die sehr hohe Komplexität der Tarifstruktur erforderte zunächst Einigkeit über die strategischen Ziele. Die Definition und Anwendung einheitlicher Tarifierungsgrundsätze ist ebenso wichtig wie die Verfügbarkeit von Spezialistenwissen im medizinischen und betriebswirtschaftlichen Bereich. Die tripartite Kooperation organisiert sich deshalb auf drei Ebenen (Abb. 1).

Fachteams werden diejenigen Arbeitsteams genannt, die entweder an übergreifenden Problemstellungen arbeiten oder direkt an konkreten Kapiteln und fachspezifischen Tarifpositionen. In den Fachteams sind insbesondere die Mitarbeit und das Wissen der medizinischen Fachgesellschaften unabdingbar. Nur so kann in den einzelnen TARMED-Kapiteln die heutige medizinische Realität möglichst korrekt abgebildet werden.

Die aus der übergeordneten Zielsetzung heraus erarbeiteten Schwerpunkte für die Gesamtrevision TARMED können wie in Tabelle 1 dargestellt gegliedert werden:

\section{Arbeiten der Fachteams im Jahr 2013}

Zur sachgerechten Abbildung der ambulanten praxis-, spital- und klinikspezifischen Leistungen haben letztes Jahr verschiedene Fachteams intensiv an ihren Tarifpositionen in den entsprechenden Kapiteln gearbeitet. Eine Übersicht über den Stand dieser Arbeiten gibt die nachfolgende Zusammenstellung:

- Fachteam «Psychiatrie»: Die Nomenklatur wurde vom Fachteam verabschiedet; es wird eine identische Systematik der Tarifstruktur für die Leistungen der delegierten Psychotherapie für die Arztpraxis und für das Spital angestrebt. 
Tabelle 1

Gliederung der Schwerpunkte der TARMED-Gesamtrevision.

\begin{tabular}{|c|c|}
\hline Schwerpunkt & Details \\
\hline $\begin{array}{l}\text { Überprüfung und Aktualisierung der } \\
\text { Kostenmodelle «KoReg», «INFRA» und } \\
\text { «Ärztliche Leistung» }\end{array}$ & $\begin{array}{l}\text { Parameter ärztliche Leistung } \\
\text { - Aktualisierung des Referenzlohns des Arztes } \\
\text { - Überprüfung der ärztlichen Produktivität } \\
\text { - Überprüfung der Abstufung des Dignitäts- } \\
\text { faktors } \\
\text { Parameter INFRA } \\
\text { - Aktualisierung der Löhne des nichtärztlichen } \\
\text { Personals } \\
\text { - Überprüfung der Personaldotation } \\
\text { - Überprüfung der Produktivität des nichtärzt- } \\
\text { lichen Personals } \\
\text { - Neubewertung der Anlagenutzungskosten } \\
\text { - Aktualisierung der Gerätepreise und Abschrei- } \\
\text { bungsdauer } \\
\text { - Überprüfung der Gerätedotation } \\
\text { - Überprüfung der Spartenauslastung } \\
\text { - Plausibilisierung des SUK-Satzes } \\
\text { Parameter KoReg } \\
\text { - Übernahme der aktuellen RoKo-Daten } \\
\text { - Überprüfung und Aktualisierung } \\
\text { der Parameter } \\
\text { - Evtl. Zusammenlegung von Sparten }\end{array}$ \\
\hline Vereinfachung des Regelwerkes & $\begin{array}{l}\text { - Vereinfachung und Reduktion auf das tari- } \\
\text { farisch Notwendige, gegebenenfalls ergänzt } \\
\text { mit Tarifstruktur externen Modulen, um } \\
\text { der Tarifstruktur die notwendige Flexibilität } \\
\text { zu geben }\end{array}$ \\
\hline Vereinfachung der Tarifstruktur & $\begin{array}{l}\text { - Weniger und transparentere Tarifpositionen } \\
\text { - Neue Struktur und Nummerierungslogik } \\
\text { - Weniger Unterebenen in den Kapiteln }\end{array}$ \\
\hline $\begin{array}{l}\text { Sachgerechte Abbildung ambulanter } \\
\text { Leistungen der Grundversorgung }\end{array}$ & $\begin{array}{l}\text { - Totale Überarbeitung der Tarifpositionen } \\
\text { im Kapitel } 00 \\
\text { - Abbildung von spezifischen Leistungen } \\
\text { für die Hausarztmedizin }\end{array}$ \\
\hline $\begin{array}{l}\text { Sachgerechte Abbildung der ambulanten } \\
\text { spital- und klinikspezifische Leistungen }\end{array}$ & $\begin{array}{l}\text { - Auf der Basis der heutigen Version } 1.08 \\
\text { TARMED, kapitelweise Revision mit Einbezug } \\
\text { der Fachgesellschaften und Spitalexperten }\end{array}$ \\
\hline $\begin{array}{l}\text { Tarifierung aller ärztlichen } \\
\text { und vermehrt auch nicht-ärztliche } \\
\text { Leistungen. Aufnahme neuer } \\
\text { medizinischer Leistungen }\end{array}$ & $\begin{array}{l}\text { - Bisher nicht tarifierte medizinische Leistungen } \\
\text { (z. B. die Kapselvideoendoskopie oder } \\
\text { nichtärztliche Leistungen) werden in die } \\
\text { Tarifstruktur integriert }\end{array}$ \\
\hline
\end{tabular}

- Fachteam «Nichtärztliche Leistungen»: Die in der heutigen Version bestehenden ca. 30 Tarifpositionen für die nichtärztlichen Leistungen (v.a. in den Kapiteln 00 und Kapitel 35) sollen, wenn immer möglich, auf einige wenige reduziert werden; zudem sollen alle nichtärztlichen Leistungen, die im ärztlichen Auftrag erbracht werden, tarifiert werden. Eine erste Version des neuen Kapitels ist ausgearbeitet und kann nun getestet werden.

- Fachteam «Ärztliche Grundleistungen»: Die mehr als 100 Tarifpositionen im Kapitel 00 sollen, wenn immer möglich, zusammengefasst werden; Auch eine Vereinfachung der Sparten (Sprechzimmer, verschiedene UBRs) in der Grundversorgung wird zusammen mit den Experten der betroffenen Fachgesellschaften diskutiert. Die speziellen
Leistungen der Hausarztmedizin sollen in einem separaten Kapitel abgebildet werden.

- Fachteam «Kardiologie»: Hauptkapitel der Kardiologie ist das Kapitel 17 mit seinen zum Teil sehr differenzierten Einzelleistungen und Zuschlagspositionen. Es wird eine Vereinfachung der Struktur angestrebt, gleichzeitig aber auch eine Tarifierung, die sich dem schnellen technischen Fortschritt in diesem Gebiet in Zukunft besser anpasst. Eine erste Tarifstruktur ist ausgearbeitet und kann im ersten Halbjahr 2014 pilotiert werden.

- Fachteam «Handchirurgie»: Tarifpositionen des Fachbereiches Handchirurgie sind heute in ganz unterschiedlichen Kapiteln vom TARMED enthalten (v.a. Kapitel 24, Kapitel 4 und 5). Auch hier, wie im Fachteam Kardiologie, wird versucht, die heutige, sehr differenzierte, aber auch stark limitierende Tarifstruktur (es gibt zum Beispiel viele «alleinige Leistungen») zu vereinfachen und transparenter zu gestalten. Die neue Nomenklatur ist weit fortgeschritten. Der Pilot, vorgesehen im 1. Semester 2014, dient einerseits der Umsetzbarkeit dieser neuen Tarifstruktur in den Kliniken und Arztpraxen, andererseits auch der Überprüfung der Minutagen.

- Fachteam «Kostenmodelle»: Dieses Fachteam widmet sich der Überprüfung, Anpassung und Aktualisierung der oben beschriebenen Parameter in den drei Kostenmodellen «KoReg», «INFRA» und «Ärztliche Leistung», die die Grundlage fast aller Tarifpositionen im TARMED bilden. Das Tarifsystem TARMED beinhaltet über alles rund 200 Parameter, die es zu prüfen und neu zu berechnen gilt. Wo möglich und verfügbar, werden aktuelle Datengrundlagen und Studien gesucht und integriert.

Auf die Dokumentation und eine plausible Argumentation wird in allen Fachteams grosser Wert gelegt, denn die geleisteten Arbeiten müssen zu einem späteren Zeitpunkt nachvollziehbar sein.

Im Sommer 2013 stellte die Technische Kommission fest, dass es viele übergeordnete (umfassende) Themenstellungen gibt, die vertieft analysiert werden müssen, da sie die Arbeiten und Neutarifierungen in den Fachteams stark beeinflussen. Seit dem Herbst 2013 arbeiten deshalb weitere Teams an der Weiterentwicklung von allgemeinen Tarifierungsgrundsätzen, insbesondere in folgenden Themenbereichen:

- Komplexität / Schwierigkeit / Dignitäten

- Abbildung Notfälle

- Kombination von Zeit- und Handlungsleistungen

- Begriff und Tarifierung von «Zugängen»

- OP-Prozess und Anästhesie-Prozess

- Tarifierung Befundung / Dokumentation / Bericht

- Wechselzeiten (Technische Grundleistungen)

- Vor- und Nachbereitung 
- Ärztliche Assistenz

- Konzept Pilotstudien

\section{Roadmap und neue Fachteams \\ im 1. Semester 2014}

Die Revisions-Arbeiten der Partner FMH, H+ und MTK werden in diesem Jahr weiter intensiviert, sowohl in den Teams bei der Weiterentwicklung der Tarifierungsgrundsätze als auch bei den Kapitelrevisionen. Folgende neue Fachteams haben ihre Facharbeiten in den letzten Wochen aufgenommen:

- Fachteam Bildgebung (Kapitel 39)

- Fachteam Pathologie (Kapitel 37)
Gemäss der gemeinsamen Roadmap (Abb. 2) ist im 1. Semester 2014 der Start weiterer Fachteams geplant. Die Intensivierung der Arbeiten wird aber nur möglich sein, wenn die personellen Ressourcen bei allen Kooperationspartnern für die Gesamtrevision noch ausgebaut werden können.

Dank der Unterstützung von Anton Prantl (Direktionspräsident der Ärztekasse) können parallel auch die TARMED-Simulationstools der FMH stetig weiterentwickelt werden. Es ist geplant, dass bald auch die Revisionspartner der FMH Gelegenheit haben werden, selbständig Simulationen via ein Web-Interface durchzuführen.

\section{Abbildung 2}

Die gemeinsame Roadmap der TARMED-Revision von H+ / FMH / MTK.

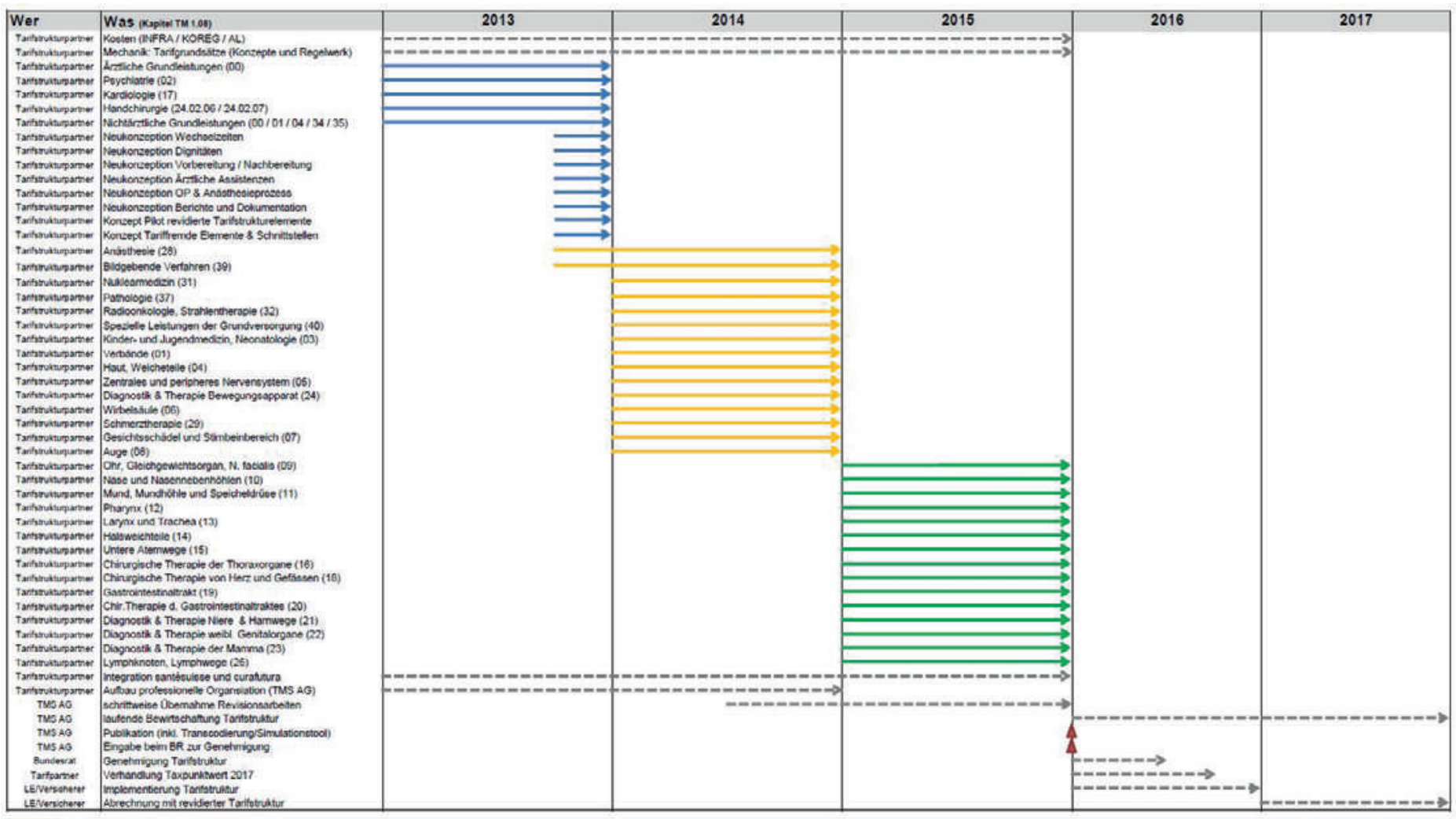

